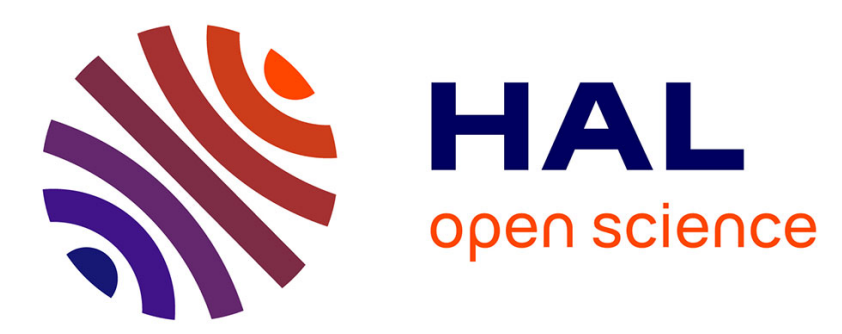

\title{
Workpiece Placement Optimization for Machining Operations with a KUKA KR270-2 Robot
}

\author{
Stéphane Caro, Claire Dumas, Sébastien Garnier, Benoît Furet
}

\section{To cite this version:}

Stéphane Caro, Claire Dumas, Sébastien Garnier, Benoît Furet. Workpiece Placement Optimization for Machining Operations with a KUKA KR270-2 Robot. 2013 IEEE International Conference on Robotics and Automation (ICRA), May 2013, Karlsruhe, France. 10.1109/ICRA.2013.6630982 . hal01726372

\section{HAL Id: hal-01726372 \\ https://hal.science/hal-01726372}

Submitted on 8 Mar 2018

HAL is a multi-disciplinary open access archive for the deposit and dissemination of scientific research documents, whether they are published or not. The documents may come from teaching and research institutions in France or abroad, or from public or private research centers.
L'archive ouverte pluridisciplinaire HAL, est destinée au dépôt et à la diffusion de documents scientifiques de niveau recherche, publiés ou non, émanant des établissements d'enseignement et de recherche français ou étrangers, des laboratoires publics ou privés. 


\title{
Workpiece Placement Optimization for Machining Operations with a KUKA KR270-2 Robot
}

\author{
Stéphane Caro ${ }^{1}$, Claire Dumas ${ }^{2}$, Sébastien Garnier ${ }^{2}$ and Benoît Furet ${ }^{2}$
}

\begin{abstract}
Roboticists are faced with new challenges in robotic-based manufacturing. Up to now manufacturing operations that require both high stiffness and accuracy have been mainly realized with computer numerical control machine tools. This paper aims to show that manufacturing finishing tasks can be performed with robotic cells knowing the process cutting conditions and the robot stiffness throughout its Cartesian workspace. It makes sense that the finishing task of large parts should be cheaper with robots. However, machining robots have not been adapted for such operations yet. As a consequence, this paper introduces a methodology that aims to determine the best placement of the workpiece to be machined knowing the elastostatic model of the robot and the cutting forces exerted on the tool. Therefore, a machining quality criterion is proposed and an optimization problem is formulated and solved. The KUKA KR270-2 robot is used as an illustrative example throughout the paper.
\end{abstract}

\section{INTRODUCTION}

Industrial robots were originally dedicated to "pick and place" operations. They start to be used for machining operations such as trimming, deflashing, degating, sanding and sawing. Machining robots are currently developed to help the operator realize machining operations, which have been mainly performed with Computer Numerical Control (CNC) machines so far. Industrial robots can be used, first to reduce scrap rates and production costs, and secondly to increase the volume and flexibility of production lines. However, industrial robots are not as accurate as CNC machine tools, but the total cost of a machining robot is $30 \%$ less compared with an equivalent $\mathrm{CNC}$ machine. Therefore, it makes sense that the larger the parts to be machined, the more interesting robot machining.

Some research works on robotic-based manufacturing have been conducted in the last two decades. The main drawback of industrial serial robots for machining operations turns to be their lack of stiffness and as a result, large manufacturing errors occur [1], [2], [3]. Indeed, a conventional machine tool is about fifty times stiffer than an industrial serial robot [2].

Matsuoka et al. [1] studied the robotic-based milling. They claimed that the machining quality can be improved by decreasing the cutting forces, namely, by decreasing the diameter of the tool and by increasing the spindle speed. Zhang et al. [2] proposed a real-time compensation of the

\footnotetext{
*This work was partially supported by the Agence Nationale de la Recherche (ANR), France (Project ANR-2010-SEGI-003- 02-COROUSSO),

${ }^{1} \mathrm{~S}$. Caro is with IRCCyN/CNRS, Nantes, France stephane.caro at irccyn.ec-nantes.fr

${ }^{2}$ C. Dumas, S. Garnier and B. Furet are with IRCCyN/University of Nantes, Nantes, France claire.dumas at univ-nantes.fr, sebastien.garnier at univ-nantes.fr, benoit.furet at univ-nantes.fr
}

robot end-effector displacement, based on the evaluation of each joint deflection. To this end, they wrote an elastostatic model of the robot at hand and used a wrench sensor to measure the forces and moments applied on the robot endeffector at any time. Besides, Tisius et al. [4] described 50 operational criteria and 50 potential criteria for serial manipulator operation, much of which applies to precision machining. Machining quality and robot energy consumption may also depend on the workpiece placement. Thus, UrRehman et al. focused on the multiobjective path placement optimization of a parallel kinematics machine based on energy consumption, shaking forces and maximum actuator torques [5].

This paper introduces a methodology that aims to determine the best placement of the workpiece to be machined knowing the cutting forces exerted on the tool and the elastostatic model of the robot. A machining quality criterion is proposed and an optimization problem is formulated and solved. The KUKA KR270-2 robot is used as an illustrative example throughout the paper.

Section 2 presents the workpiece test and the cutting forces applied by the workpiece on the tool along the machined path. Section 3 describes the robotic cell. Section 4 deals with the formulation of a mono-objective optimization problem to find the optimum placement of the workpiece with regard to a proposed machining quality criterion. Section 5 highlights the optimum and worst workpiece placements within the robotic cell. The best and worst redundancy planning schemes associated with those placements are also determined. Section 6 is about the conclusions of the paper and the future work.

\section{MACHINING TASK}

Figure 1 illustrates the workpiece to be machined, which is made up of aluminum alloy. $\mathcal{F}_{W}$ of origin $O_{W}$ is the frame attached to the workpiece. The five segments $A B$, $B C, C O_{W}, O_{W} D$ and $D E$, of length equal to $200 \mathrm{~mm}$ each, have to be milled. The tool path is offset by the tool radius from the five segments to be milled. The tool path is discretized into $n$ points and is shown in dashed line in Fig. 1. Frame $\mathcal{F}_{P i}$ is attached to the $i$ th point of the tool path, $i=1, \ldots, n . X_{P i}$ is along the feed direction. $Z_{P i}$ is along the tool axis and points toward the robot.

The machining quality is affected by the robot deviation due to the cutting forces applied on the tool [6]. The cutting conditions are given in Tab. I where $f_{z}, a_{p}$ and $a_{e}$ denote the feed rate, the depth of cut and the width of cut, respectively. The cutting forces are evaluated thanks to the cutting force 


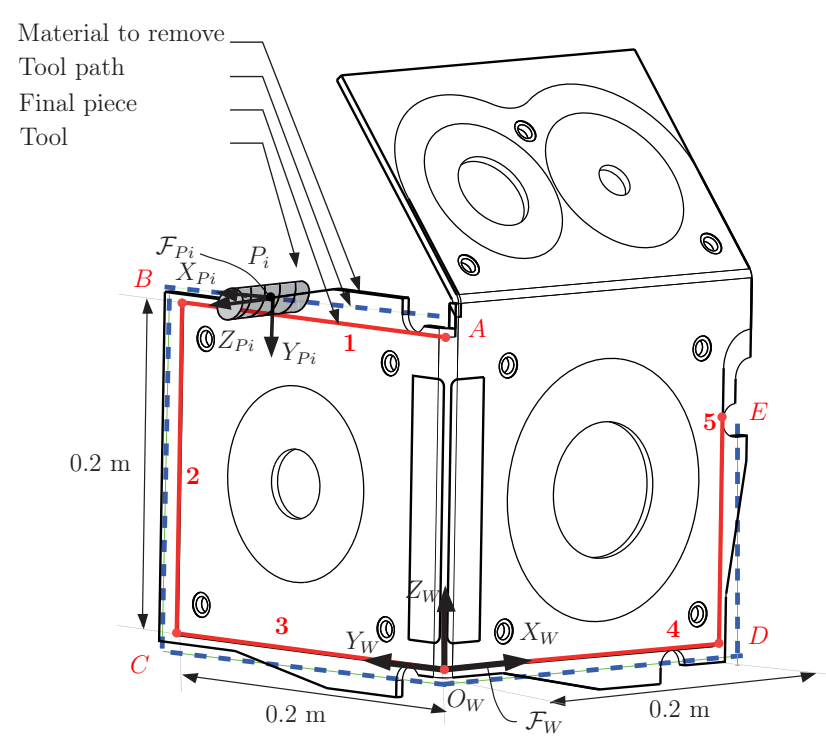

Fig. 1. Workpiece

TABLE I

CUTTING Conditions

\begin{tabular}{ccccc}
\hline \hline Spindle speed & Feed speed & $f_{z}$ & $a_{p}$ & $a_{e}$ \\
$20000 \mathrm{rpm}$ & $4 \mathrm{~m} / \mathrm{min}$ & $0.05 \mathrm{~mm} /$ tooth $/ \mathrm{rev}$ & $5 \mathrm{~mm}$ & varies \\
\hline \hline
\end{tabular}

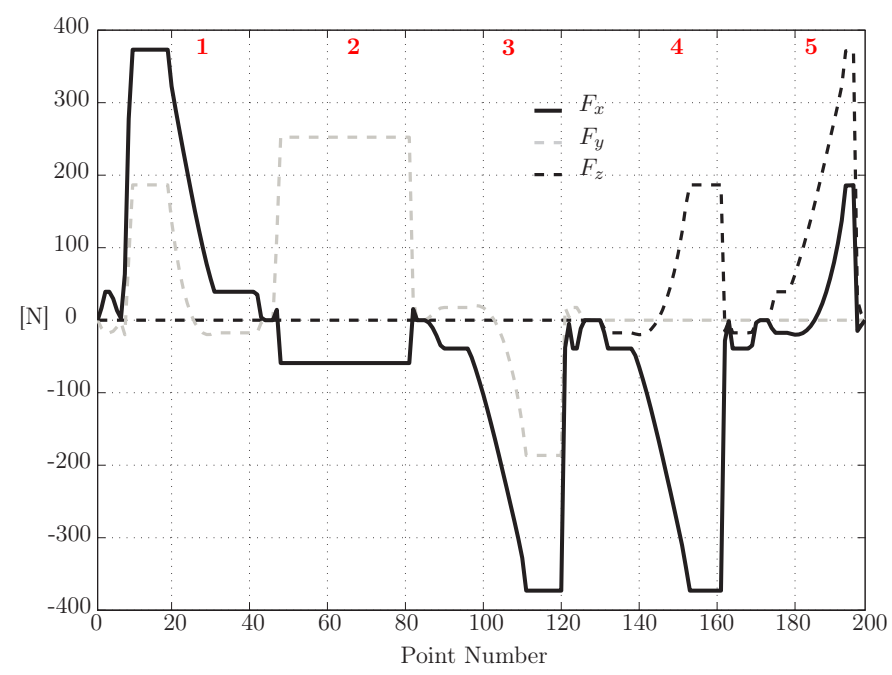

Fig. 2. Force components applied by the tool on the workpiece expressed in frame $\mathcal{F}_{W}$ as a function of the tool path point number

model described in [7]. As a result, Fig. 2 depicts the force components applied by the tool on the workpiece along axes $X_{W}, Y_{W}$ and $Z_{W}$ of frame $\mathcal{F}_{W}$ as a function of the tool path point number.

\section{ROBOTIC CELL AND PARAMETERIZATION}

The robotic cell shown in Fig. 3 is composed of a KUKA KR270-2 robot, a FISCHER milling spindle mounted on the robot end-effector and a rotary table.

The geometric parameters of the robot are presented in [8]. An identification procedure was developed in [9] in order to determine all joint stiffness values of the KUKA

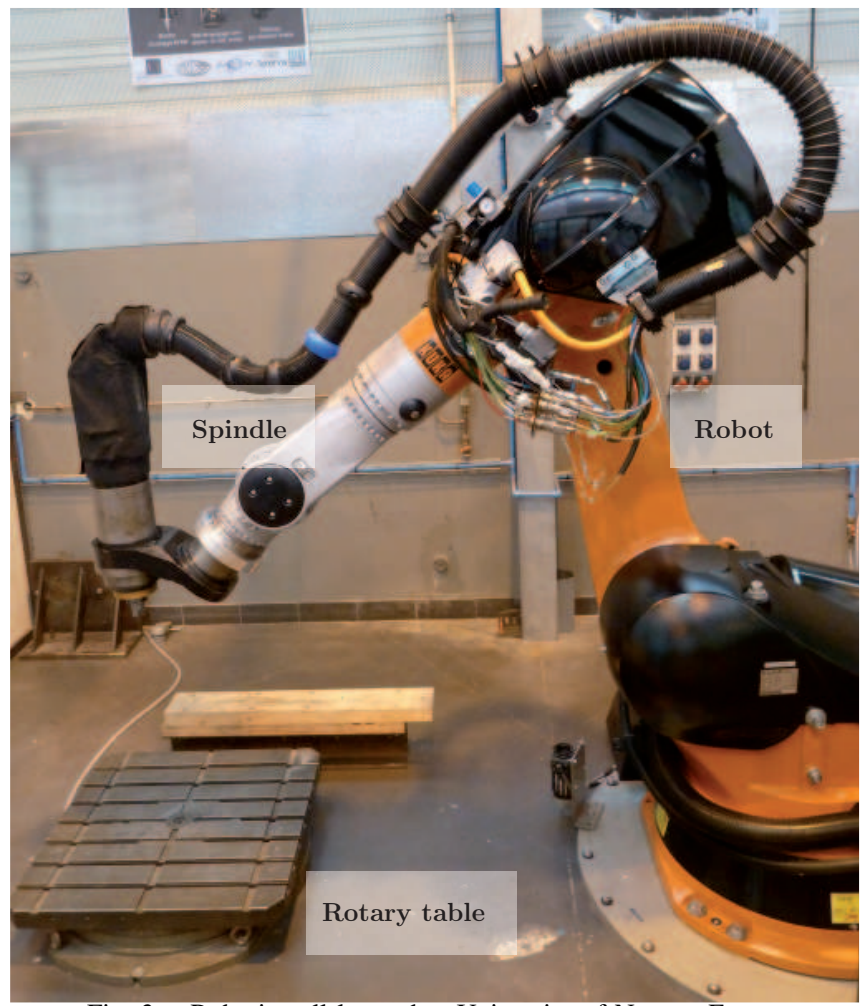

Fig. 3. Robotic cell located at University of Nantes, France

KR270-2 robot. Accordingly, the translational and rotational displacements of the tool can be predicted knowing the wrench exerted on it. It is noteworthy that the flexibility of the links is considered through the identified joint stiffness values.

TABLE II

JOINT STIFFNESS VALUES OF KUKA KR270-2 RoBOT IN [MNM/RAD]

\begin{tabular}{cccccc}
\hline \hline$k_{\theta_{1}}$ & $k_{\theta_{2}}$ & $k_{\theta_{3}}$ & $k_{\theta_{4}}$ & $k_{\theta_{5}}$ & $k_{\theta_{6}}$ \\
0.237 & 3.32 & 2.79 & 0.486 & 0.521 & 0.38 \\
\hline \hline
\end{tabular}

The joint stiffness values of the KUKA KR270-2 robot are given in Tab. II $k_{\theta_{l}}$ is the $l$ th joint stiffness value, $l=$ $1, \ldots, 6$.

The FISCHER milling spindle is of type MFW 1709/24. Its maximum rotational speed is equal to $24000 \mathrm{rpm}$ and its power is equal to $20 \mathrm{~kW}$.

The orientation of the workpiece can be fully represented with the variables $\left(Q_{2}, Q_{3}, Q_{4}\right)$, a subset of the quaternions coordinates. Indeed, the quaternions represent the rotations of the workpiece with a rotation axis $\mathbf{u}=\left[\begin{array}{lll}u_{x} & u_{y} & u_{z}\end{array}\right]^{T}$ and an angle $\theta$. The relation between the quaternions and the axis and angle representation can be found in [10]:

$$
\begin{aligned}
& Q_{1}=\cos (\theta / 2) \\
& Q_{2}=u_{x} \sin (\theta / 2) \\
& Q_{3}=u_{y} \sin (\theta / 2) \\
& Q_{4}=u_{z} \sin (\theta / 2)
\end{aligned}
$$


where $u_{x}^{2}+u_{y}^{2}+u_{z}^{2}=1$ and $0 \leq \theta \leq \pi$.

Nevertheless, the workpiece can be only rotated about the vertical axis of the robot base frame $\mathcal{F}_{0}$ due to the geometry of the rotary table. Therefore,

$$
Q_{2}=Q_{3}=0
$$

and

$$
-1 \leq Q_{4} \leq 1
$$

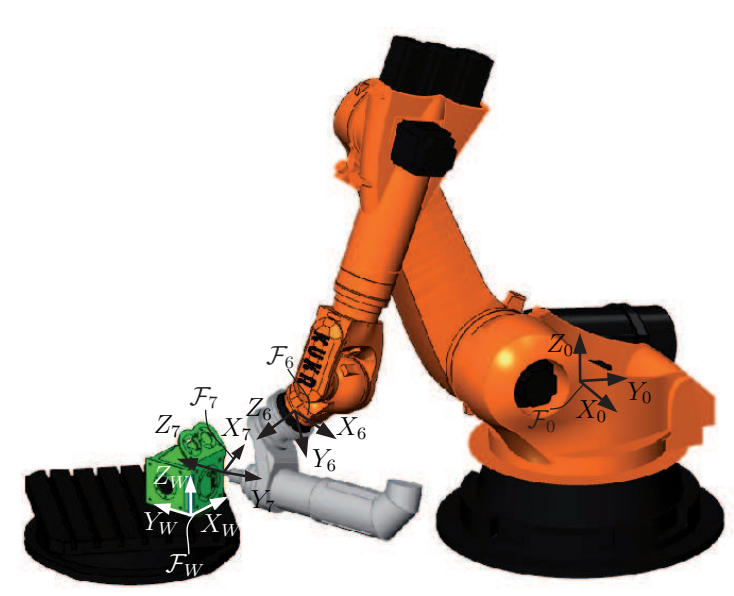

Fig. 4. Closed loop chain and frames $\mathcal{F}_{0}, \mathcal{F}_{6}, \mathcal{F}_{7}$ and $\mathcal{F}_{W}$

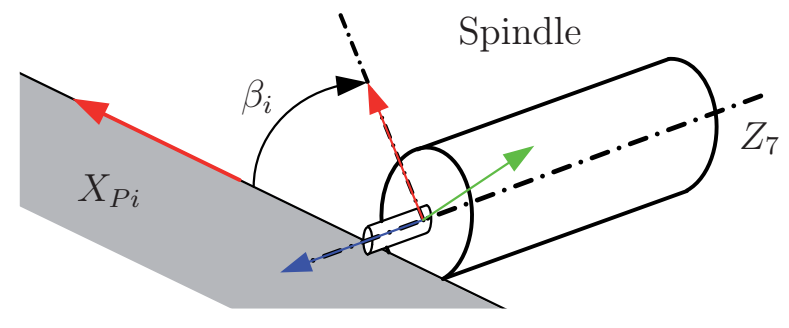

Fig. 5. Kinematic redundancy characterized with angle $\beta_{i}$

Figure 4 represents the closed loop chain composed of the KUKA KR270-2 robot, the FISCHER milling spindle, the workpiece and the rotary table. It also depicts the robot base frame $\mathcal{F}_{0}$, the frame $\mathcal{F}_{6}$ attached to the robot end-effector, the frame $\mathcal{F}_{7}$ attached to the spindle and the workpiece frame $\mathcal{F}_{W}$. From the previous closed loop chain, we can write:

$$
{ }^{0} T_{W}{ }^{W} T_{P i}={ }^{0} T_{6}{ }^{6} T_{7}{ }^{7} T_{P i}
$$

where

${ }^{0} T_{W}$ is the homogeneous transformation matrix from frame $\mathcal{F}_{0}$ to frame $\mathcal{F}_{W}$ expressed as:

$$
{ }^{0} T_{W}=\left[\begin{array}{cccc}
2 Q_{1}^{2}-1 & -2 Q_{1} Q_{4} & 0 & { }^{0} x_{O W} \\
Q_{1} Q_{4} & 2 Q_{1}^{2}-1 & 0 & { }^{0} y_{O W} \\
0 & 0 & 1 & { }^{0} z_{O W} \\
0 & 0 & 0 & 1
\end{array}\right]
$$

${ }^{0} x_{O W},{ }^{0} y_{O W}$ and ${ }^{0} z_{O W}$ being the Cartesian coordinates of point $O_{W}$ expressed in frame $\mathcal{F}_{0}$.

${ }^{W} T_{P i}$ is the homogeneous transformation matrix from frame $\mathcal{F}_{W}$ to frame $\mathcal{F}_{P i}$ attached to the $i$ th point of the tool path as shown in Fig. 1
${ }^{0} T_{6} \quad$ is the homogeneous transformation matrix from frame $\mathcal{F}_{0}$ to frame $\mathcal{F}_{6}$.

${ }^{6} T_{7}$ is the homogeneous transformation matrix from frame $\mathcal{F}_{6}$ to frame $\mathcal{F}_{7}$ and depends on the geometry of the spindle and how the latter is mounted on the robot end-effector. Here,

$$
{ }^{6} T_{7}=\left[\begin{array}{cccc}
0 & -\sqrt{2} / 2 & \sqrt{2} / 2 & 0 \\
1 & 0 & 0 & 0 \\
0 & \sqrt{2} / 2 & \sqrt{2} / 2 & 0.684 \\
0 & 0 & 0 & 1
\end{array}\right]
$$

${ }^{7} T_{P i}$ is the homogeneous transformation matrix from frame $\mathcal{F}_{P i}$ to frame $\mathcal{F}_{7}$. Note that the KUKA KR270-2 robot has six degrees of freedom, whereas the milling operation sets only five degrees of freedom as the rotation of the spindle about the tool axis is not fixed. Therefore, the robot is redundant with respect to the task and the kinematic redundancy is equal to one as explained in [11], [12]. Here, the kinematic redundancy is characterized by the angle $\beta_{i}$, which corresponds to the rotation angle of the spindle about the tool axis at the $i$ th point of the tool path as shown in Fig. 5. As a consequence,

$$
{ }^{7} T_{P i}=\left[\begin{array}{cccc}
\cos \left(\beta_{i}\right) & \sin \left(\beta_{i}\right) & 0 & 0 \\
\sin \left(\beta_{i}\right) & -\cos \left(\beta_{i}\right) & 0 & 0 \\
0 & 0 & -1 & 0 \\
0 & 0 & 0 & 1
\end{array}\right]
$$

\section{OPTIMUM WORKPIECE PLACEMENT}

This section deals with a methodology that aims to determine the best placement of the workpiece to be machined knowing the cutting forces exerted on the tool and the elastostatic model of the robot. The proposed methodology is highlighted through the definition of a machining quality criterion and the formulation of a mono-objective optimization problem.

\section{A. Machining quality criterion}

It makes sense that the tool displacement $c_{i}$, normal to both the feed direction along axis $X_{P i}$ and the tool axis $Z_{7}$, is mainly responsible for machining errors. ${ }^{0} \mathbf{x}_{P i}$ and ${ }^{0} \mathbf{z}_{7}$ are the unit vectors of axes $X_{P i}$ and $Z_{7}$ expressed in frame $\mathcal{F}_{0}$, respectively. $c_{i}$ can be expressed as follows:

$$
c_{i}=\left|{ }^{0} \delta \mathbf{d}_{i}^{T}\left({ }^{0} \mathbf{z}_{7} \times{ }^{0} \mathbf{x}_{P i}\right)\right|
$$

where ${ }^{0} \delta \mathbf{d}_{i}$ is the point-displacement of the tool at the $i$ th point of the tool path expressed in frame $\mathcal{F}_{0}$ and $\times$ denotes the cross product. The point-displacement ${ }^{0} \delta \mathbf{d}_{i}$ is evaluated thanks to the Cartesian stiffness matrix $\mathbf{K}$ of the KUKA KR270-2 robot obtained in [8] and from the wrench $\mathbf{w}$ applied by the tool on the workpiece that are depicted in Fig. 2, namely,

$$
{ }^{0} \delta \mathbf{d}_{i}=\mathbf{K}^{-1} \mathbf{w}
$$


The machining quality criterion $f_{M Q C}$, which is the objective function of the optimization problem at hand, is defined as the mean of $c_{i}$ values, namely,

$$
f_{M Q C}=\frac{1}{n} \sum_{i=1}^{n} c_{i} \rightarrow \min
$$

The smaller $f_{M Q C}$, the better the machining quality.

\section{B. Decision variables}

The decision variable vector of the optimization problem at hand contains the Cartesian coordinates of point $O_{W}$ expressed in frame $\mathcal{F}_{0}$, i.e., ${ }^{0} x_{\mathrm{OW}},{ }^{0} y_{\mathrm{OW}}$ and ${ }^{0} z_{\mathrm{OW}}$ and the orientation parameter $Q_{4}$ of the workpiece.

The decision variable vector also contains the redundancy planning scheme. Let $\beta_{i}$ be the rotation angle of the spindle about the tool axis at the $i$ th point of the discretized tool path as shown in Fig. 5 . Accordingly, the redundancy planning scheme is defined by vector $\boldsymbol{\beta}$ expressed as:

$$
\boldsymbol{\beta}=\left[\begin{array}{llll}
\beta_{1} & \beta_{2} & \cdots & \beta_{n}
\end{array}\right]^{T}
$$

with $-\pi \leq \beta_{i} \leq \pi, i=1, \ldots, n$.

The KUKA KR270-2 robot may have eight solutions to the inverse geometric model, namely, eight postures. The robot has to keep the same posture along a given segment in order to avoid signs of wear. However, the robot posture can change from one segment to the next one. $\mu_{j}$ denotes the solution number to the inverse geometric model of the robot along the $j$ th segment of the tool path, $j=1, \ldots, 5$. As a consequence, the posture scheme is defined by vector $\boldsymbol{\mu}$ expressed as:

$$
\boldsymbol{\mu}=\left[\begin{array}{lllll}
\mu_{1} & \mu_{2} & \mu_{3} & \mu_{4} & \mu_{5}
\end{array}\right]^{T}
$$

with $\mu_{j} \in[1, . ., 8]$.

\section{Constraints}

Four types of constraints arise in the optimization problem at hand:

1) The joint angles of the KUKA KR270-2 robot should be bounded between its joint limits,

$$
\begin{aligned}
-185 \mathrm{deg} & \leq \theta_{1 i} \leq 185 \mathrm{deg} \\
0 & \leq \theta_{2 i} \leq 146 \mathrm{deg} \\
-245 \mathrm{deg} & \leq \theta_{3 i} \leq 29 \mathrm{deg} \\
-350 \mathrm{deg} & \leq \theta_{4 i} \leq 350 \mathrm{deg} \\
-125 \mathrm{deg} & \leq \theta_{5 i} \leq 125 \mathrm{deg} \\
-350 \mathrm{deg} & \leq \theta_{6 i} \leq 350 \mathrm{deg}
\end{aligned}
$$

$\theta_{j i}$ being the $j$ th joint angle of the robot, $j=1, \ldots, 6$, when the tool is located at the $i$ th point of the tool path, $i=1, \ldots, n$. Note that the robot was parameterized by using the modified Denavit-Hartenberg convention [10].

2) The tool displacement $c_{i}$ expressed with (11) should be smaller than a given value $c_{\max }$, which is defined with regard to the expected machining quality, i.e.,

$$
\max \left(\left|c_{1}\right|,\left|c_{2}\right|, \cdots,\left|c_{n}\right|\right) \leq c_{\max }
$$

where |.| denotes the absolute value.

3) The workpiece should be located within a work volume that depends on the rotary table size and location within the robotic cell. Here,

$$
\begin{aligned}
& x_{\min } \leq^{0} x_{O W} \leq x_{\max } \\
& y_{\min } \leq^{0} y_{O W} \leq y_{\max } \\
& z_{\min } \leq^{0} z_{O W} \leq z_{\max }
\end{aligned}
$$

with $x_{\min }=-0.3 \mathrm{~m}, x_{\max }=0.3 \mathrm{~m}, y_{\min }=$ $-2.24 \mathrm{~m}, y_{\max }=-1.64 \mathrm{~m}, z_{\min }=-0.602 \mathrm{~m}$ and $z_{\max }=-0.214 \mathrm{~m}$.

4) The robot should be far from singularities while the tool follows the path, namely,

$$
1 / \kappa_{F}\left(\mathbf{J}_{N i}\right)>0.2, \quad i=1, \ldots, n
$$

where $\mathbf{J}_{N i}$ is the normalized kinematic Jacobian matrix of the KUKA KR270-2 robot defined in [8] and evaluated at the $i$ th point of the tool path. $\kappa_{F}\left(\mathbf{J}_{N i}\right)$ is condition number of matrix $\mathbf{J}_{N i}$ based on the Frobenius norm.

\section{Formulation of the optimization problem}

From (13) to (26), the optimization problem to solve in order to find the best workpiece placement can be formulated as follows:

$$
\begin{aligned}
\text { minimize } & f_{M Q C} \\
\text { over } & \mathbf{x}=\left[{ }^{0} x_{O W}{ }^{0} y_{O W}{ }^{0} z_{O W} Q_{4} \boldsymbol{\beta}^{T} \boldsymbol{\mu}^{T}\right]^{T} \\
\text { subject to } & \max \left(\left|c_{1}\right|,\left|c_{2}\right|, \cdots,\left|c_{n}\right|\right) \leq c_{\max } \\
& -185 \mathrm{deg} \leq \theta_{1 i} \leq 185 \mathrm{deg} \\
& 0 \leq \theta_{2 i} \leq 146 \mathrm{deg} \\
& -245 \mathrm{deg} \leq \theta_{3 i} \leq 29 \mathrm{deg} \\
& -350 \mathrm{deg} \leq \theta_{4 i} \leq 350 \mathrm{deg} \\
& -125 \mathrm{deg} \leq \theta_{5 i} \leq 125 \mathrm{deg} \\
& -350 \mathrm{deg} \leq \theta_{6 i} \leq 350 \mathrm{deg} \\
& 1 / \kappa_{F}\left(\mathbf{J}_{N i}\right)>0.2 \\
& x_{\min } \leq{ }^{0} x_{O W} \leq x_{\max } \\
& y_{\min } \leq{ }^{0} y_{O W} \leq y_{\max } \\
& z_{\min } \leq{ }^{0} z_{O W} \leq z_{\max } \\
& -1 \leq Q_{4} \leq 1 \\
& i=1, \ldots, n
\end{aligned}
$$

Optimization problem 27) aims to find the optimum workpiece placement and the corresponding optimum redundancy planning scheme that minimize the machining quality criterion $f_{M Q C}$ defined by (13) while respecting the set of constraints.

\section{RESULT ANALYSIS}

A hybrid optimization algorithm was used to solve optimization problem 27. As a matter of fact, a genetic algorithm [13] was used to find the initial guess of an 


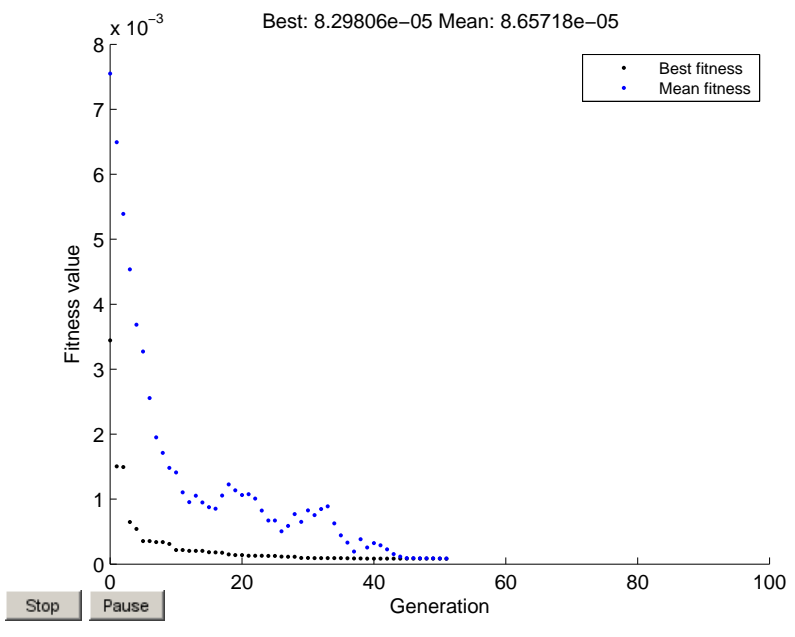

Fig. 6. Convergence of the genetic algorithm for the determination of the best workpiece placement

interior-point algorithm for large-scale nonlinear programming [14]. The Matlab ga function combined with the Matlab fmincon function were used.

The genetic algorithm converged after 51 generations as shown in Fig. 6, each population containing 120 individuals. Then, a local optimum decision variable vector $\mathbf{x}_{\text {opt }}$ of optimization problem (27) was obtained with the Matlab fmincon function and is expressed as:

$\mathbf{x}_{\text {opt }}=\left[\begin{array}{llllll}0.0022 & -1.6435 & -0.5564 & 0.9258 & \boldsymbol{\beta}_{\text {opt }} & \boldsymbol{\mu}_{\text {opt }}\end{array}\right]$

with $\boldsymbol{\mu}_{\text {opt }}=\left[\begin{array}{lllll}4 & 4 & 4 & 5 & 5\end{array}\right]^{T}$ meaning that the robot posture changes between segment 3 and segment 4 only.

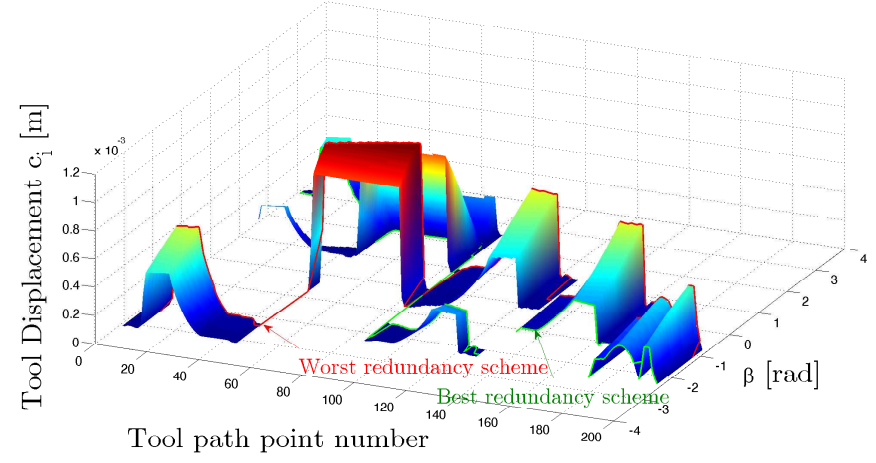

Fig. 7. Tool displacement $c_{i}[\mathrm{~m}]$ as a function of the tool path point number and $\beta$ angle for the best workpiece placement. The green curve characterizes the optimum redundancy planning scheme, i.e., $\boldsymbol{\beta}_{\text {opt }}$ vector, whereas the red curve represents the worst redundancy planning scheme for this workpiece placement

Figure 7 illustrates the tool displacement $c_{i}$ with respect to the tool path point number and $\beta$ angle for the optimal workpiece placement. The green curve characterizes the optimum redundancy planning scheme, i.e., $\boldsymbol{\beta}_{\text {opt }}$ vector, whereas the red curve represents the worst redundancy planning scheme for this workpiece placement. The white areas can not be reached by the robot because of its joint limits.
It is noteworthy that ${ }^{0} x_{O W},{ }^{0} y_{O W},{ }^{0} z_{O W}, Q_{4}$ and $\boldsymbol{\mu}$ are the only decision variables considered by the $g a$ and fmincon functions in this optimization problem solving. As a matter of fact, an optimal $\boldsymbol{\beta}$ vector is searched at each iteration of the genetic algorithm and at each iteration of the interior-point algorithm. This vector is obtained in such a way that it minimizes the objective function $f_{M Q C}$ and avoids discontinuities in the robot joint space along each segment.

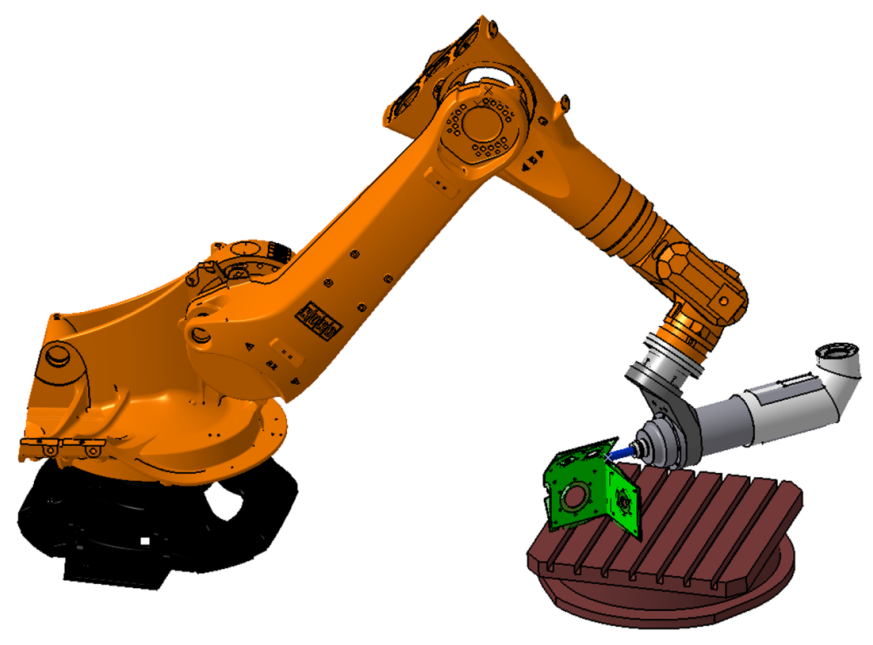

Fig. 8. Optimum workpiece placement

${ }_{7}^{T}$ Figure 8 represents the optimum workpiece placement found by solving optimization problem (27).

It is noteworthy that the worst workpiece placement is obtained by maximizing the objective function $f_{M Q C}$ while respecting the constraints of optimization problem [27].

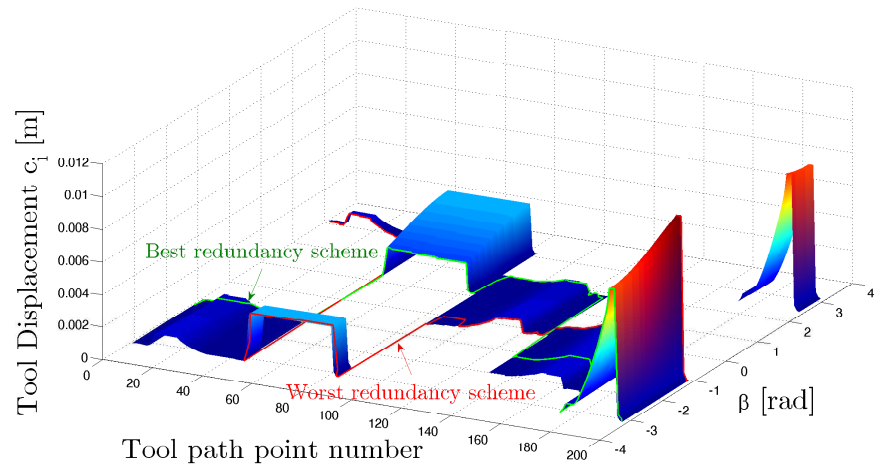

Fig. 9. Tool displacement $c_{i}[\mathrm{~m}]$ as a function of the tool path point number and $\beta$ angle for the worst workpiece placement. The green curve characterizes the optimum redundancy planning scheme, whereas the red curve represents the worst redundancy planning scheme for this workpiece placement

Figure 9 illustrates the tool displacement $c_{i}$ with respect to the tool path point number and $\beta$ angle for the worst workpiece placement. The green curve characterizes the optimum redundancy planning scheme, i.e., $\boldsymbol{\beta}_{\text {opt }}$ vector, whereas the red curve represents the worst redundancy planning scheme for this workpiece placement. 
Let us compare the machining quality of the workpiece obtained with the four following milling conditions:

Case 1: Optimum workpiece placement with the best redundancy planning scheme (see Fig. 7)

Case 2: Optimum workpiece placement with the worst redundancy planning scheme (see Fig. 7p

Case 3: Worst workpiece placement with the best redundancy planning scheme (see Fig. 91

Case 4: Worst workpiece placement with the worst redundancy planning scheme (see Fig. 9)

An video of the milling operation corresponding to Case 1 can be downloaded a 11 An video of the milling operation corresponding to Case 2 can be downloaded a $2^{2}$ An video of the milling operation corresponding to Case 3 can be downloaded $\mathrm{a}^{3}$ An video of the milling operation corresponding to Case 4 can be downloaded at

TABLE III

MACHINING QUALITy CRITERION FOR Four MILLING CONDITIONS

\begin{tabular}{ccccc}
\hline \hline & Case 1 & Case 2 & Case 3 & Case 4 \\
$f_{M Q C}[\mathrm{~mm}]$ & 0.083 & 0.35 & 0.82 & 1.2 \\
\hline \hline
\end{tabular}

Table III gives the values of the maching quality criterion $f_{M Q C}$ defined in 13 for the previous four milling conditions. It is apparent that both the workpiece placement and the kinematic redundancy affect the machining quality of the workpiece. Table III shows that it is important to pay attention to the workpiece placement and kinematic redundancy. Indeed, $f_{M Q C}$ value for Case 4 is more than 14 times higher than $f_{M Q C}$ value for Case 1.

\section{CONCLUSIONS AND FUTURE WORK}

This paper introduced a methodology to determine the optimum placement of any workpiece to be machined knowing the cutting forces exerted on the tool and the elastostatic model of the KUKA KR270-2 robot. First, a workpiece test was introduced and the forces applied by the workpiece to the tool were evaluated by using an existing cutting force model. Then, the robotic cell was presented. It contains a KUKA KR270-2 robot, a FISCHER milling spindle and a rotary table. The parameterization of the closed loop chain composed of the foregoing components and the workpiece was also described. A machining quality criterion was introduced and a mono-objective optimization problem was formulated in order to determine the optimum placement of a workpiece to be machined and the associated best redundancy planning scheme. The worst workpiece placement and the associated worst redundancy planning were also obtained by maximizing the objective function of the optimization

${ }^{1}$ http://www.irccyn.ec-nantes.fr/ caro/ICRA2013/video1.avi

${ }^{2}$ http://www.irccyn.ec-nantes.fr/ caro/ICRA2013/video2.avi

${ }^{3}$ http://www.irccyn.ec-nantes.fr/ caro/ICRA2013/video3.avi

${ }^{4}$ http://www.irccyn.ec-nantes.fr/ caro/ICRA2013/video4.avi

5Please, be sure that the tilde " " symbol before "caro" is kept when the previous four links are copied and pasted in your web browser for downloading the videos. problem at hand. It is noteworthy that the machining quality criterion corresponding to the optimum workpiece placement and the associated best redundancy planning scheme is more than 14 times smaller than the machining quality criterion corresponding to the worst workpiece placement and the associated worst redundancy planning scheme. Therefore, it is important to pay attention to the workpiece placement and kinematic redundancy for machining operations with a KUKA KR270-2 robot.

The theoretical results presented in this paper will be checked experimentally soon. The extension of the proposed methodology to robot machining operations with a higher kinematic redundancy of the robot with regard to the task, such as a machining robot mounted on a rail or a mobile platform, are also part of the future work.

\section{ACKNOWLEDGMENT}

The authors would like to acknowledge the financial support of the ANR, France (Project ANR-2010-SEGI-00302-COROUSSO).

\section{REFERENCES}

[1] Matsuoka, S.-I., Shimizu, K., Yamazaki, N. and Oki, Y. (1999). "HighSpeed End Milling of an Articulated Robot and its Characteristics," Elsevier, Journal of Materials Processing Technology, 95, pp. 83-89.

[2] Zhang, H., Hang, H., Wang, J., Zhang, G., Gan, Z., Pan, Z., Cui, H. and Zhu, Z. (2005). "Machining with Flexible Manipulator: Toward Improving Robotic Machining Performance," Proceedings of the 2005 IEEE/ASME International Conference on Advanced Intelligent Mechatronics, Monterey, California, USA, 24-28 July.

[3] Abele, E., Weigold, M. and Rothenbcher, S. (2007). "Modeling and Identification of an Industrial Robot for Machining Applications," Elsevier, Annals of the CIRP, 56/1/2007.

[4] Tisius, M., Pryor, M., Kapoor, C. and Tesar, D. (2009). "An Empirical Approach to Performance Criteria for Manipulation," ASME, Journal of Mechanisms and Robotics, 1(3), pp. 031002-1-031002-12.

[5] Ur-Rehman, R., Caro, S., Chablat, D. and Wenger, P. (2010). "Multiobjective Path Placement Optimization of Parallel Kinematics Machines Based on Energy Consumption, Shaking Forces and Maximum Actuators Torques: Application to the Orthoglide," Mechanism and Machine Theory, 45, pp. 1125-1141.

[6] Dumas, C., Caro, S., Garnier, S. and Furet, B. (2012). "Workpiece Placement optimization of Six-revolute Industrial Serial Robots for Machining Operations," The ASME/ESDA 2012 International Conference on Engineering Systems Design and Analysis (ESDA 2012), Nantes, France, July 2-4.

[7] Tlusty, J. and Macneil, P. (1975). "Dynamics of cutting forces in end milling," Annals of the CIRP, 24(1), pp. 21-25.

[8] Dumas, C., Caro, S., Cherif, M., Garnier, S. and Furet, B. (2011). "Joint Stiffness Identification of Industrial Serial Robots," Robotica, Available on CJO 2011 doi:10.1017/S0263574711000932.

[9] Dumas, C., Caro, S., Garnier, S. and Furet, B. (2011). "Joint Stiffness Identification of Six revolute Industrial Serial Robots," Robotics and Computer Integrated Manufacturing, 27(4), pp. 881-888.

[10] Khalil, W. and Dombre, E. (2002). Modeling, Identification and Control of Robots, Hermes Penton Ltd.

[11] Conkur, E.S. and Buckingham, R. (1997). "Clarifying the definition of redundancy as used in robotics," Robotica, 15, pp. 583-586..

[12] Wenger, P. (2004). "Curve-following for redundant manipulators with obstacles : feasibility analysis and solutions," Journal IFToMM Problems of Applied Mechanics, 24(1), No.2, pp. 17-26.

[13] Goldberg, D.E. (1989). Genetic Algorithms in Search, Optimization \& Machine Learning, Addison-Wesley.

[14] Byrd, R.H., Mary, E.H. and Nocedal, J (1999). "An Interior Poin Algorithm for Large-Scale Nonlinear Programming," SIAM Journal on Optimization, 9(4), pp. 877-900. 